\title{
Atypical Presentation of Orbital Natural Killer cell Lymphoma
}

\author{
Brandon Menke* \\ University of South Carolina, Columbia \\ *Corresponding author: Brandon Menke, University of South Carolina, 9 Medical Park, Suite 340, Columbia, SC 29202

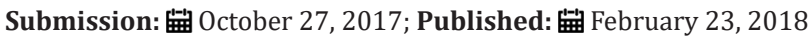

\begin{abstract}
Natural killer (NK)/T-cell lymphoma is a rare malignancy accounting for less than 1 percent of all lymphomas in North America and Europe. Ocular manifestations frequently presented as orbital cellulites that does not improve with adequate antibiotics. We report a case of NK/T cell lymphoma with rare ocular manifestation that was initially confused with orbital cellulites and chronic sinusitis due to multiple negative biopsies. 92-year-old female presented with right eye vision loss, and ipsi lateral orbital swelling for four weeks. MRI demonstrated right intra-orbital extension to the orbital apex. Workups for infection and vasculitis were negative. Multiple biopsies with histopathology and flow cytology were unrevealing for malignancy. Patient failed to improve on antibiotics and steroids. Five months after the initial presentation, patient presented with the same complaint; however, the orbital mass had grown in size and involved the maxillary sinus and contra lateral side. Re-biopsy revealed positive CD 56 for NK lymphoma.
\end{abstract}

\section{Introduction}

Natural killer (NK)/T-cell lymphoma is a rare primary nonHodgkin lymphoma. It can be divided into nasal, non-nasal, and aggressive NK leukaemia/lymphoma subtypes [1]. Nasal NK/T cell lymphoma refers to malignancies originating in the nasopharyngeal area, causing destruction of the nasal cavity, paranasal sinuses, orbits, and surrounding structures. Common presenting signs are midline mass with obstruction, nasal bleeding, and perforation of the hard palate [3]. This subtype is more common in Asia and South America and relatively rare in Western countries [2]. The average age of affected patients is 50-60 years old, and it occurs three times more in males than females [3]. Due to its rare incidence and deceiving presentation, it is often misdiagnosed as chronic sinusitis, granuloma, or fungal infection; thus, the final diagnosis and treatments can be delayed.

The majority of patients with nasal NK lymphoma are positive for Epstein Barr virus (EBV). In fact, EBV viral load has been used in disease staging [1]. Microscopically, nasal NK lymphoma is marked by aggressive angioinvasion in a background of CD3+ and CD56+ atypical lymphocytes and inflammatory cells [3]. The prognosis is relatively poor with a 5-year cumulative survival rate of approximately 40\% [4]. CHOP regimen (cyclophosphamide, doxorubicin, vincristine and prednisolone) is used concurrently with radiotherapy for stage 1 and 2, while chemotherapy alone is the mainstay of treatment for stages 3 and 4 [5]. Autologous or allogeneic hematopoietic stem cell transplantation has been considered in advanced disease with unclear prognostic benefits
[5]. This report presents the first case of an elderly Caucasian female with NK lymphoma initially presenting as orbital cellulites. Collection and evaluation of protected patient health information was HIPAA-compliant.

\section{Case Presentation}

A 92-year-old Caucasian female with history of hypothyroidism, glaucoma, migraines, cataracts, and hypertension was admitted to the hospital with a one-week history of right eye vision loss that was preceded by ipsilateral swelling and associated bilateral pain for four weeks. Examination revealed an afferent pupillary defect and abduction deficit of right eye, partial ptosis and proptosis, no pain with ocular movements, and no drainage or significant swelling or abnormality on slit lamp exam. On dilated fundo scopic exam, optic nerve cup to disc ratios were 0.3 with sharp nerve margins bilaterally; macula, vessels, and peripheral retina appeared intact. Uncorrected visual acuities were hand motion on the right, and $20 / 30$ on the left eye. Intraocular pressures were 19 on the right, and 13 on the left. Lab results showed WBC 6.32, $\mathrm{Hb}$ 15.3, platelets 189, ESR 11, CRP 11.1. MRA revealed multifocal atherosclerosis with no aneurysm or occlusion. MRI demonstrated a right intra-orbital infiltrate along the medial wall with extension to the orbital apex causing optic nerve and medial rectus muscle impingement and complete opacification/consolidation of the right ethmoid air cells and sphenoid sinuses (Figure 1). Orbital cellulites was suspected at this point. The patient was started on aggressive antibiotics and taken for sinus drainage and medial 
orbitotomy. Endoscopy observed semi solid and granular whitish material in ethmoid sinuses, extensive mucus in the sphenoid sinus, and no bony destruction. Several biopsies were taken of the sinus demonstrating inflamed stoma with mixed serous and mucinous glands, dense inflammatory infiltrate of acute and chronic inflammatory cells with areas of necrosis, rare eosinophils and benign respiratory epithelium. Cultures and special stains for bacteria and fungi were positive only for Propionibacterium acne. The leading differential diagnosis at this point was chronic sinusitis with necrosis. Patient was discharged on rapid prednisone taper and 4 weeks of vancomycin /ceftriaxone /metronidazole.

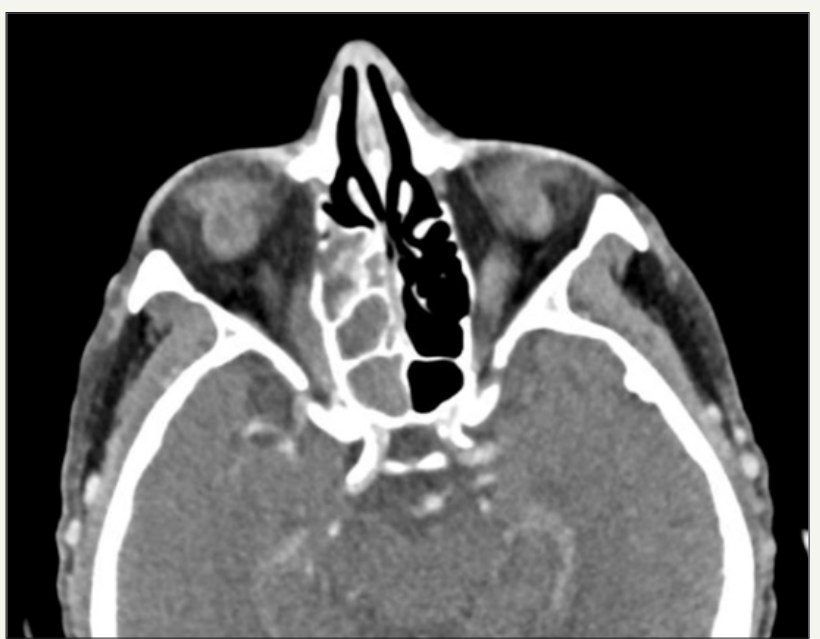

Figure 1 : Abduction deficit, partial ptosis, and proptosis of the right eye (top: straight gaze, middle: left gaze, bottom: right gaze)

The patient was readmitted 2 weeks later for lower extremity deep vein thrombosis in the setting of a thrombocytopenia of 65000 and a persistent medial orbital mass. A new antibiotic regimen of doxycycline/metronidazole resulted in resolution of the thrombocytopenia. Antibiotics were later discontinued all together due to lack of ocular improvement. The patient was taken back to the operating room for a repeat biopsy due to a CT scan showing an enhancing mass involving the extraconal soft tissue paralleling the posterior ethmoid air cells, concerning for a possible neoplasm. Intra operatively, the medial rectus appeared to be grayish in color concerning for muscle infarction. Final pathology noted a partial fibro vascular and fibro muscular necrosis containing only a few crushed lymphocytes and scattered acute inflammatory cells. Cultures continued to be negative. Flow cytometry was negative for lymphoma. No diagnostic evidence of malignancy or features of lymph proliferative disorder were indicated on histology. CT chest/ abdomen/pelvis to rule out metastatic malignancy was only notable for a $1 \mathrm{~cm}$ pulmonary nodule in left lower lobe. Idiopathic orbital inflammatory syndrome labs showed mildly elevated erythrocyte sedimentation rate and CRP; negative RPR, ANA, c-ANCA and p-ANCA. Given these findings, the diagnosis of chronic sinusitis with orbital extension was still the leading diagnosis. The patient was discharged again on a steroid taper (Figures $2 \& 3$ ).

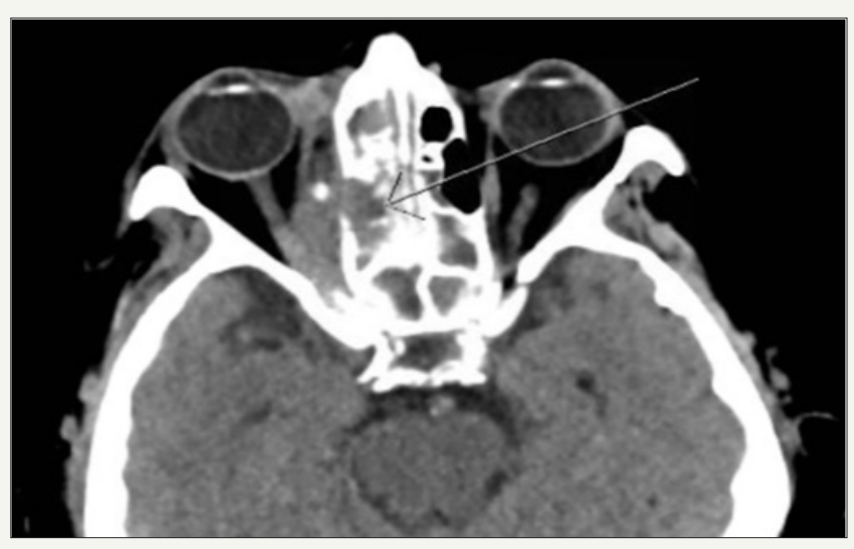

Figure 2 : Retrobulbar extraconal mass along the medial orbit near the apex impinging on the right optic nerve, and consolidation in the ethmoid air cells and the sphenoid sinus

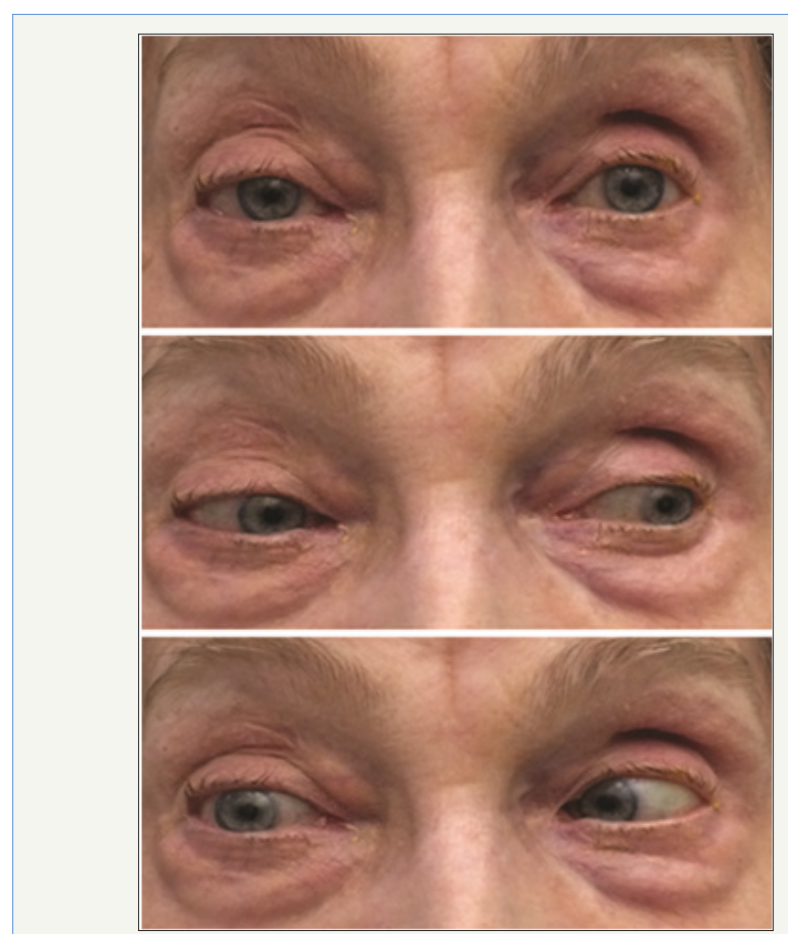

Figure 3 : Persistent opacification of sphenoid sinuses, right ethmoidal air cells with extension from medial orbit into the orbial apex (arrow). New involvement of maxiallary and possible frontal sinuses

month later, the patient presented for the third time after sustaining a fall with accompanying fever. On examination, complete ophthalmoplegia and complete ptosis of the right side with V1-V2 anaesthesia consistent with orbital apex syndrome was identified. CT sinus showed that the orbital apex mass had increased in size and now involved the maxillary sinus and the contra lateral side. The patient also endorsed intermittent fever, night sweats, and 6 pounds of weight loss over the past month. She was transferred to a tertiary care facility for further workup. There, chest X-ray and CT head were again negative. She initially did well with medial wall orbital decompression and biopsy and was started on antifungal 
and antibiotics regimens, but subsequently developed altered mental status. Due to her deteriorating condition, the patient and family declined further interventions and instead opted for home palliative care. The biopsy results were positive for CD56 consistent with the diagnosis of NK lymphoma.

\section{Discussion}

Natural killer (NK)/T-cell lymphoma is a rare malignancy accounting for less than 1 percent of all lymphomas in North America and Europe [6]. The nasal type involves the nose and sinuses with possible orbital extension. Common presenting signs are midline mass with obstruction, nasal bleeding, and perforation of the hard palate [3]. Ocular manifestations are infrequently reported [7]. However, if orbital extension does occur, it may be the first sign of disease and present as orbital cellulitis that does not improve with adequate antibiotics [8]. Imaging may demonstrate bony destruction and a soft tissue mass invading surrounding structures [9]. However, these findings are nonspecific and can indicate systemic granulomatous disease or local tumours. Histologically, NKTL shows polymorphic inflammatory infiltration with angio destruction. However, in early stages, atypical cells are scarce and obscured by inflammatory cells recruited from tumour secreting cytokines, which can lead to a misdiagnosis of inflammatory processes and infection [10]. EBV, CD56, and CD3 are strongly associated with NKTL and have been used to define NKTL in published case series [11]

Our case demonstrates a rare presentation of NK/T lymphoma mimicking orbital cellulites and chronic sinusitis. This 92-year-old Caucasian female patient was not part of the typical demographic which is a 50-year-old Asian male. There was no nasal obstruction, ulcer, or epitasis as frequently described in literature. The patient's slight improvement on antibiotics and steroid initially gave false reassurance of the diagnosis of cellulites. Furthermore, over the course of 4 months, multiple tissue biopsies and flow cytometry performed specifically to rule out malignancy were negative.

Clinicians must maintain a high index of suspicion and have NKTL in their differential diagnoses in patients that appear to have persistent orbital cellulitis despite antibiotics, especially when orbital apex signs are present without significant pain. Initial negative immune histo chemistry and histology cannot be used to completely rule out this type of lymphoma due to a lack of sensitivity. Bony destruction in the context of what appears to be cellulites or sinusitis should raise suspicion of malignancy and as in this case, multiple biopsies may be required to make the diagnosis.

\section{References}

1. Kwong YL (2005) Natural killer-cell malignancies: diagnosis and treatment. Leukemia 19(12): 2186-2194.

2. Ishida F, Kwong YL (2010) Diagnosis and management of natural killercell malignancies. Expert Rev Hematol 3(5): 593-602.

3. Kwong YL (2011) The diagnosis and management of extranodal NK/Tcell lymphoma, nasal-type and aggressive NK-cell leukemia. J Clin Exp Hematop 51(1): 21-28.

4. Vose J, Armitage J, Weisenburger D (2008) International peripheral T-cell and natural killer/T-cell lymphoma study: pathology findings and clinical outcomes. J Clin Oncol 26(25): 4124-4130.

5. Coha B, Vucinic I, Mahovne I, Vukovic-arar Z (2014) Extranodal lymphomas of head and neck with emphasis on NK/T-cell lymphoma, nasal type. J Craniomaxillofac Surg 42(2): 149-152.

6. Vega F, Lin P, Medeiros LJ (2005) Extranodal lymphomas of the head and neck. Ann Diagn Pathol 9(6): 340-350.

7. Chen CS, Miller NR, Lane A, Eberhart C (2008) Third cranial nerve palsy caused by intracranial extension of a sino-orbital natural killer T-cell lymphoma. J Neuroophthalmol 28(1): 31-35.

8. Kim JW, An JH (2014) Extranodal natural killer/T-cell lymphoma, nasal type, of the orbit mimicking recurrent orbital cellulitis. J Craniofac Surg 25(2): 509-511.

9. King AD, Lei KI, Ahuja AT, Lam WW, Metreweli C (2000) MR imaging of nasal T-cell/natural killer cell lymphoma. Am J Roentgenol 174(1): 209211.

10. Kawakami K, Ito R, Tono Y, Yazaki A, Murata T, et al. (2012) Orbital inflammatory lesion as an initial manifestation of systemic nasal type NK/T-cell lymphoma. J Clin Exp Hematop 52(2): 137-139.

11. Hon C, Kwok AK, Shek TW, Chim JC, Au WY, et al. (2002) Visionthreatening complications of nasal T/NK lymphoma. Am J Ophthalmol 134(3): 406-410.

\section{Your subsequent submission with Crimson Publishers will attain the below benefits}

- High-level peer review and editorial services

- Freely accessible online immediately upon publication

- Authors retain the copyright to their work

- Licensing it under a Creative Commons license

- Visibility through different online platforms

- Global attainment for your research

- Article availability in different formats (Pdf, E-pub, Full Text)

- Endless customer service

- Reasonable Membership services

- Reprints availability upon request

- One step article tracking system 